\title{
Distribuição espaço-temporal e sucesso reprodutivo de Eretmochelys imbricata nas praias do Ipojuca, Pernambuco, Brasil
}

\author{
Carina C. de M. Moura ${ }^{1,2}$, Elisângela da S. Guimarães ${ }^{1,2}$, Geraldo J. B. de Moura ${ }^{1,2}$, Getúlio J. A. do \\ Amaral $^{2,3} \&$ Arley C. da Silva ${ }^{2}$ \\ 1. Universidade Federal Rural de Pernambuco, Laboratório de Herpetologia e Paleoherpetologia, Rua Dom Manoel de Medeiros, s/n, 52171-900, Dois Irmãos, PE, Brasil. (carinacarneiro1@gmail.com) \\ 2. ONG Ecoassociados, km 0, Galeria Espaço Arena, sala 03, Ipojuca, PE, Brasil. (www.ecoassociados.org) \\ 3. Universidade Federal de Pernambuco, Departamento de Estatística, Av. Prof. Moraes Rego, 1235, Cidade Universitária, 50670-901, Recife, PE, Brasil.
}

\begin{abstract}
Spatio-temporal distribution and reproductive success of Eretmochelys imbricata on the beaches of Ipojuca, Pernambuco, Brazil. This study aimed to verify the spatio-temporal distribution of Eretmochelys imbricata (Linnaeus, 1766) and aspects of its reproductive biology, such as incubation time, reproductive success, biometric measurements of females, number of nests and fecundity. Data were collected during 2007 to 2010, on the beaches of Muro Alto, Cupe, Merepe, Porto de Galinhas, and Maracaípe, all of them located in the city of Ipojuca, state of Pernambuco, Brazil. Parameters relating to reproductive biology and nesting areas of the species were comparatively analyzed. Eretmochelys imbricata was recorded nesting between October and May, when 350 nests were monitored through three seasons. The spawning peak happened from January to March, also revealing a seasonal pattern. The number of nests differed significantly between seasons. The Merepe beach presented an elevated occurrence of nests $(46 \mathrm{nests} / \mathrm{km})$ if compared to the other monitored beaches. On the aspects of reproductive biology, the reproductive success was $65,6 \%$ and the incubation time interval ranged from 54 to 56 days. Biometric measurements were collected from 59 specimens, resulting in an average of $92,5 \mathrm{~cm} \pm 4,5$ for the curved carapace length, and of $83,4 \mathrm{~cm} \pm 5$ for the curved carapace width. The results can be used for subsidize conservation plans and demonstrate that the beaches recorded in this study are relevant as nesting areas for $E$. imbricata.
\end{abstract}

KEYWORDS. Hawksbill turtle, seasons, reproduction, ecology, Testudine.

RESUMO. Este estudo objetivou verificar a distribuição temporal e espacial de Eretmochelys imbricata (Linnaeus, 1766) e aspectos de sua biologia reprodutiva, tais como tempo de incubação, sucesso reprodutivo, biometria das fêmeas, número de ninhos e fecundidade. Os dados foram coletados de 2007 a 2010 nas praias de Muro Alto, Cupe, Merepe, Porto de Galinhas e Maracaípe, todas elas localizadas no município do Ipojuca, estado de Pernambuco, Brasil. Foram analisados comparativamente parâmetros relativos à biologia reprodutiva e áreas de nidificação da espécie. Eretmochelys imbricata foi registrada nidificando entre os meses de outubro a maio, totalizando 350 ninhos monitorados em três temporadas. Os picos de desova ocorreram de janeiro a março, revelando um padrão sazonal das desovas. Houve diferença significativa entre o número de ninhos nas temporadas. A praia de Merepe apresentou uma ocorrência elevada de ninhos ( 46 ninhos $/ \mathrm{km}$ ) em relação às demais praias monitoradas. Quanto aos aspectos da biologia reprodutiva, o sucesso reprodutivo foi $65,6 \%$, e o intervalo do tempo de incubação de 54 a 56 dias. As medidas biométricas foram coletadas de 59 espécimes, e apresentaram média de $92,5 \mathrm{~cm} \pm 4,5$ para o comprimento curvilíneo da carapaça e de $83,4 \mathrm{~cm}$ \pm 5 para a largura curvilínea da carapaça. Os resultados podem ser utilizados para subsidiar planos de conservação e demonstram que as praias registradas neste estudo têm relevância como áreas de nidificação para E. imbricata.

PALAVRAS-CHAVE. Tartaruga-de-pente, temporadas, reprodução, ecologia, Testudine.

As tartarugas marinhas são sauropsidas ectotérmicos, pertencentes ao clado Testudine (RAPHAEL, 2003) que, no processo reprodutivo, realizam sazonalmente migrações das áreas de forrageamento para as áreas de acasalamento (MilLER, 1997). Os fatores determinantes da distribuição espacial e temporal, no período reprodutivo, estão relacionados, principalmente, com aspectos abióticos como temperatura, granulometria (Ferreira JR, 2009), umidade (PACKARD et al., 1987; Miller, 1997) e a declividade praial (Mortimer, 1990).

No Brasil, E. imbricata apresenta registros de nidificação entre os estados do Piauí (SANTANa et al., 2009) e do Espírito Santo (Gomes et al., 2007). As áreas de reprodução consideradas prioritárias para esta espécie estão localizadas no norte do estado da Bahia (Marcovaldi et al., 1999), Sergipe e no litoral sul do Rio Grande do Norte (Marcovaldi et al., 2011). No nordeste brasileiro, o período de nidificação ocorre entre os meses de novembro a abril (MARCOVALDi et al., 2011), tendo picos nos meses de dezembro a fevereiro na Bahia (CAMillo et al., 2009) e em Sergipe (Marcovaldi et al., 2011). Na Paraíba, a temporada reprodutiva apresenta picos em fevereiro e março (Mascarenhas et al., 2003), períodos caracterizados por apresentarem temperaturas elevadas, menores pluviosidade e umidade relativa (INMET, $3^{\circ}$ Distrito).

A biologia reprodutiva das tartarugas marinhas vem sendo estudada a partir do número de ovos por ninhada, número de ninhos, tempo de incubação, sucesso de eclosão, número de filhotes, biometria (MARCOVALDI et al., 1999; XAVIER et al., 2006), e de dados relativos ao ciclo migratório, como intervalo internidal e intervalo de remigração, os quais são muito úteis para estudos sobre comportamento reprodutivo (Alvarado \& Murphy, 1999). Entretanto, os dois últimos só podem ser quantificados mediante marcação das tartarugas marinhas (Spotila, 2004; López-Mendilaharsu \& Rосна, 2009).

Investigar aspectos ecológicos como distribuição e biologia reprodutiva de E. imbricata que nidifica no litoral Sul de Pernambuco é essencial para subsidiar estratégias de proteção e manejo (MARCovaldi et al., 1999; Ansos et al., 2004), fornecendo dados relevantes para auxiliar na identificação de novas áreas de nidificação (Marcovaldi \& Marcovaldi, 1999; Spotila, 2004). 
Assim objetivamos, através do monitoramento das praias de nidificação de Eretmochelys imbricata no município do Ipojuca, litoral sul de Pernambuco, verificar como ocorre a distribuição espaço-temporal e observar aspectos relativos à biologia reprodutiva desta espécie durante as temporadas reprodutivas de 2007 a 2010. Este artigo poderá subsidiar projetos de conservação de E. imbricata, que está classificada como criticamente ameaçada de extinção (IBAMA, 2003; IUCN, 2010; CITES, 2011).

\section{MATERIAL E MÉTODOS}

Área de estudo. A área de estudo localizase no município do Ipojuca, situado na mesorregião metropolitana e na microrregião Suape do Estado de Pernambuco, Brasil (08 24'25'S; 35 03'45'W). Do total de $32 \mathrm{~km}$ de área litorânea, $12 \mathrm{~km}$ foram monitorados ao longo das praias de Muro Alto, Cupe, Merepe, Porto de Galinhas e Maracaípe.

A praia de Muro Alto, com aproximadamente 2,5 $\mathrm{km}$, está margeada por recifes de arenito e limita-se ao norte pelo Porto de Suape e ao sul pela praia de Cupe; esta apresenta extensão de $2,37 \mathrm{~km}$, sendo aparente a presença de um pequeno banco recifal. A praia Merepe é a maior em extensão, com $3,47 \mathrm{~km}$; não apresenta barreiras de acesso à praia e está ligada à praia de Porto de Galinhas, que possui $1,47 \mathrm{~km}$ e é um dos principais pontos turísticos do estado, tendo por isso muita interferência antrópica, também margeada por recifes de corais. A praia Maracaipe, com 3,11 km e próxima a uma região de estuário, apresenta recifes de arenito margeando a costa de forma irregular. Todas as praias possuem trechos com vegetação e influência direta de condomínios, hotéis e pousadas.

Amostragem. A metodologia é baseada na utilizada pelo Projeto TAMAR - ICMBio (MARCOVALDI \& MARCOVALDI, 1999).

Foram realizadas rondas noturnas, no horário da maré mais baixa, feitas com o auxílio de um veículo motorizado, durante três temporadas reprodutivas consecutivas, nos meses de outubro a junho dos anos 2007/2008, 2008/2009 e 2009/2010. Os ninhos foram registrados tanto pelo flagrante das fêmeas no momento da desova, quanto pela visualização do rastro deixado pelas tartarugas na areia. Para menor interferência sobre o processo de desova, desligava-se o veículo e o grupo esperava o término da oviposição.

As medidas biométricas da carapaça de indivíduos adultos foram coletadas logo após o término da postura e fechamento do ninho, no retorno das fêmeas ao mar. Para as medições do comprimento curvilíneo da carapaça (CCC) e largura curvilínea da carapaça (LCC), utilizouse fita métrica com precisão de $0,1 \mathrm{~cm}$. O comprimento foi medido desde o início da placa pré-central até o fim da placa pós-central, e a largura mensurada a partir da porção mais larga da carapaça, pela maior distância entre placas marginais correspondentes (MARCOVALDI \& Marcovaldi, 1985; Bolten, 1999; Marcovaldi \& Marcovaldi, 1999; Wyneken, 2001).

Depois de confirmada as desovas, pela localização das câmaras de ovos, os ninhos foram cercados com estacas e fita zebrada, isolando-os para evitar o tráfego de pessoas, pisoteio e compactação da areia (PRITCHARD \& Mortimer, 1999). Os ninhos foram monitorados até o dia da emergência dos neonatos, período que corresponde a uma estimativa do tempo de incubação. Cada ninho foi aberto dois ou três dias após a emergência, sendo feita contagem das cascas dos ovos para estimar o número de filhotes vivos, dos filhotes que completaram seu desenvolvimento porém não sobreviveram (natimortos) e dos ovos não eclodidos (Miller, 1997).

Análise de dados. Para avaliar aspectos da biologia reprodutiva e distribuição espaço-temporal, foram realizadas análises comparativas de três temporadas reprodutivas.

As análises estatísticas foram conduzidas com a linguagem de programação R (R Development CoRE TEAm, 2009). Como os procedimentos utilizados para as análises não estão diretamente disponíveis nesta linguagem de programação, foram desenvolvidos algoritmos para realização das mesmas.

A fecundidade, a proporção do número de vivos e o tempo de incubação das três temporadas reprodutivas foram analisados; para tal foi utilizado o teste não paramétrico Kruskal-Wallis (KRUSKAL \& Wallis, 1952). Para verificar se existem grupos de indivíduos com características similares foi usado o método de agrupamento Kmeans aplicando o algoritmo HARTIGAN \& WONG (1979), que busca encontrar centros de agrupamentos naturais nos dados.

Em relação ao tempo de incubação, as análises foram feitas com o objetivo de se obter um intervalo de confiança e os percentis da distribuição empírica utilizando o método de Bootstrap (DAVISON \& HINCKLEY, 1997). Utilizou-se o modelo SARIMA, parâmetro autoregressivo de média móvel para verificar se existe sazonalidade nos dados analisados (WERNER \& RIBEIRO, 2003), a partir deste modelo avaliou-se a variável número de ninhos/mês, para realizar esta análise, é necessária uma amostra que exceda 50 dados. Assim, os dados de 2004 a 2007 foram cedidos pela ONG Ecoassociados, sendo amostrados 72 meses (sete temporadas reprodutivas consecutivas, de 2004 a 2010) nesta análise. Este modelo é ideal para analisar a distribuição sazonal das tartarugas marinhas, podendo ser usado como controle para verificar se em um determinado mês houve diferença entre o número de desovas observadas e o número esperado, ou se aquele número previsto pelo modelo ocorreu.

\section{RESULTADOS}

Ao longo das três temporadas reprodutivas foram registrados e monitorados um total de 350 ninhos de 
E. imbricata nas cinco praias estudadas. Paralelamente registrou-se seis ninhos de outras espécies: quatro de Caretta caretta (Linnaeus, 1758) e dois de Lepidochelys olivacea (Eschscholtz, 1829).

Distribuição temporal. As tartarugas de pente iniciam as desovas no mês de outubro, estendendo-se até o início de maio. Registramos a maior parte dos ninhos $(83,5 \%)$ nos meses de janeiro, fevereiro e março, meses caracterizados pelas elevadas temperaturas médias (INMET, $3^{\circ}$ Distrito). Em 2007/2008 e 2008/2009 observou-se uma tendência a um maior número de desovas nos meses de fevereiro e março, enquanto que na temporada 2009/2010 os meses mais numerosos foram janeiro e fevereiro (Fig. 1).

Tendo-se em vista que a série de desovas apresenta um comportamento sazonal, pode-se perceber que há um padrão de repetição das autocorrelações significativas, o que indica sazonalidade (Fig. 2).
Observou-se que há padrão sazonal durante o período de desovas de tartarugas de pente (Fig. 3). O modelo obtido serve para análise, controle e previsão. Por exemplo, no caso de controle percebe-se que o sexagésimo mês apresentou um valor bem superior aquele previsto pelo modelo, o que provavelmente seja provocado por flutuações aleatórias da população.

Houve um aumento no número de ninhos registrados de 2007/2008 para 2008/2009, de 100 ninhos para 153. Entretanto, houve um decréscimo de 32,6\% em 2009/2010 com 103 ninhos registrados (Fig. 4).

Distribuição espacial. Considerando os dados das três temporadas, foi possível observar maior ocorrência de ninhos na praia Merepe $(50 \%, \mathrm{~N}=179)$, seguida pela praia do Cupe $(23 \%, \mathrm{~N}=83)$ e Muro Alto $(13 \%$, $\mathrm{N}=46$ ) (Fig. 5). Os menores valores foram registrados em Maracaípe $(10 \%, \mathrm{~N}=36)$ e Porto de Galinhas $(3 \%$, $\mathrm{N}=10)$ (Tab. I).

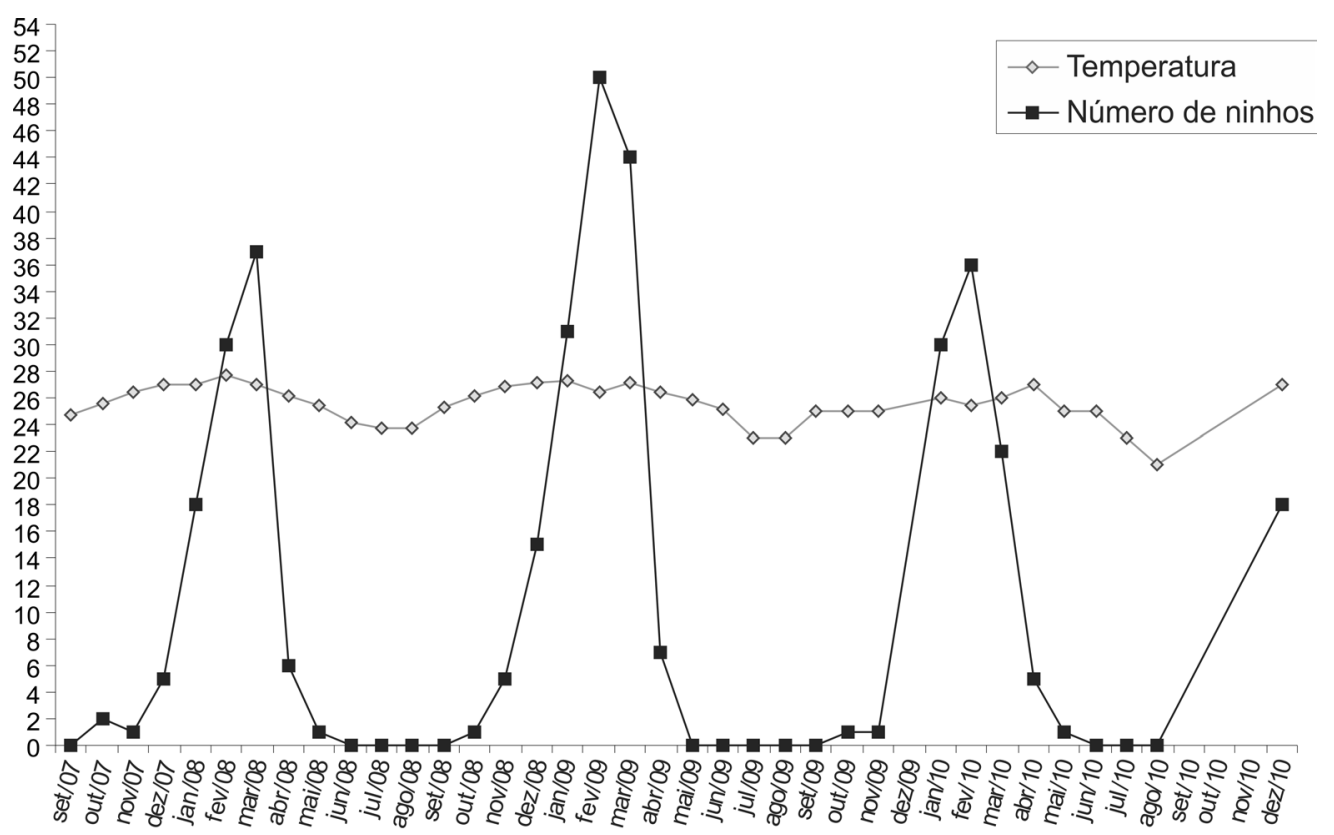

Fig. 1. Picos das desovas de Eretmochelys imbricata (Linnaeus, 1766) no município de Ipojuca, PE, Brasil entre os meses de janeiro a março das três temporadas reprodutivas, meses que apresentam temperaturas mais elevadas.

\section{Função de Autocorrelação}

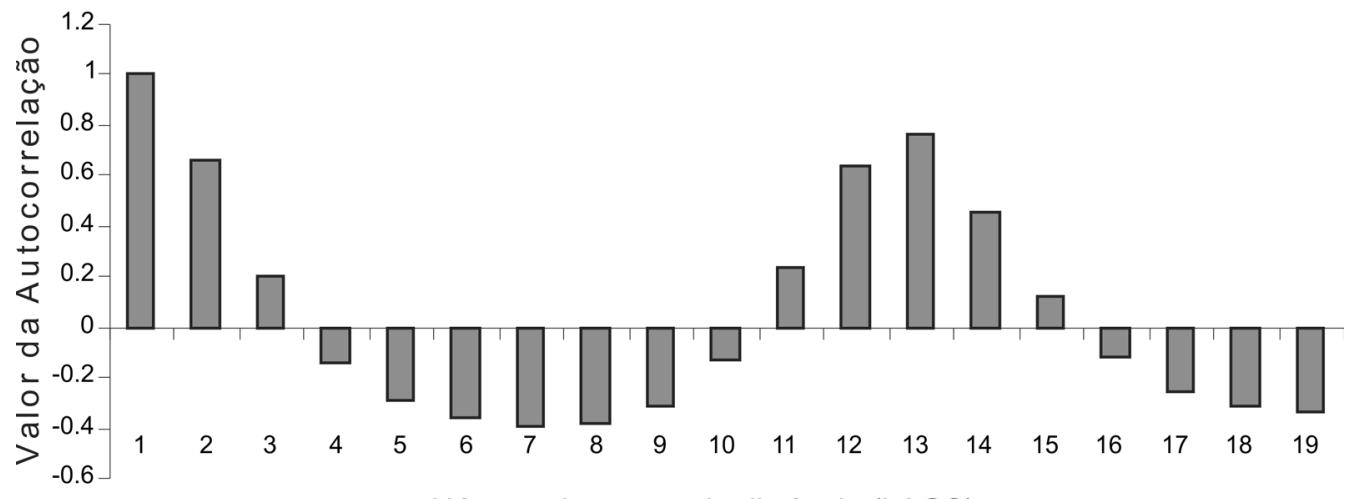

Número de meses de distância (LAGS)

Fig. 2. Função de autocorrelação da série temporal de desovas de Eretmochelys imbricata (Linnaeus, 1766) no município de Ipojuca, PE, Brasil. Pode-se verificar uma repetição nas autocorrelações, sugerindo sazonalidade para a série de desovas. 
A frequência de ninhos nas praias foi de aproximadamente 30 ninhos $/ \mathrm{km}$. A maior frequência ocorreu na praia Merepe, com 46 ninhos $/ \mathrm{km}$. A praia de Muro Alto teve 39 ninhos/km, Cupe 35, Maracaípe 12 e Porto de Galinhas 7 ninhos $/ \mathrm{km}$. A maior frequência de

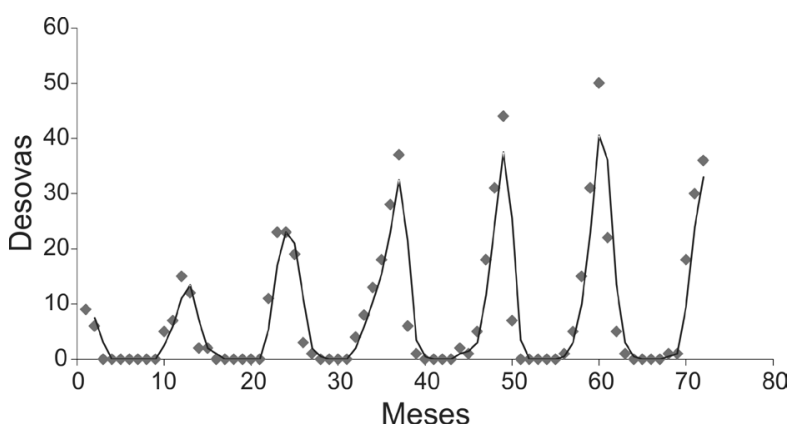

Fig. 3. Sazonalidade das desovas de tartarugas-de-pente Eretmochelys imbricata (Linnaeus, 1766) durante 72 meses no município de Ipojuca, PE, Brasil. Modelo SARIMA (2,0,3),(3,1,2). Número Mensal de Desovas (pontos) e Valor Ajustado pelo modelo (linha).

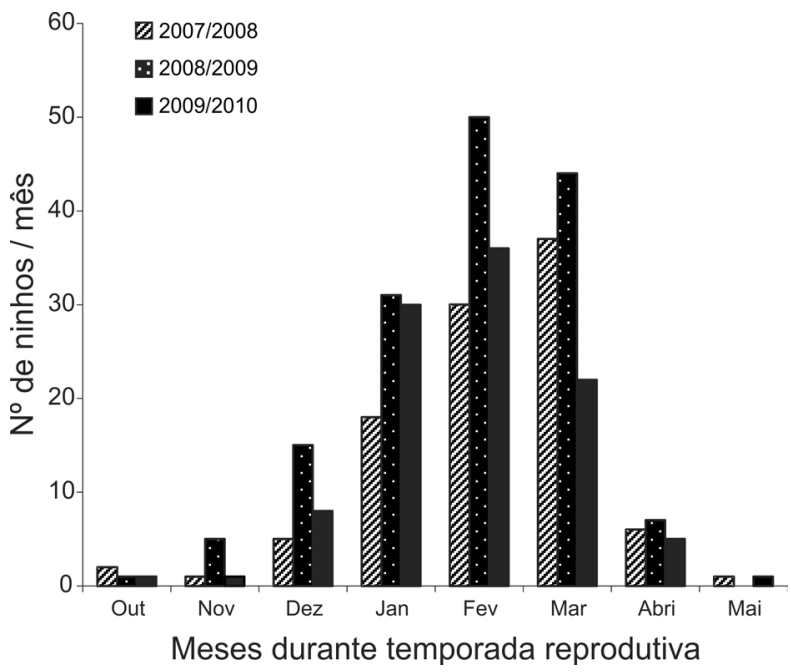

Fig. 4. Número de ninhos/mês de Eretmochelys imbricata (Linnaeus, 1766) nas praias de Ipojuca, PE, Brasil durante as temporadas reprodutivas de 2007 a 2010 .

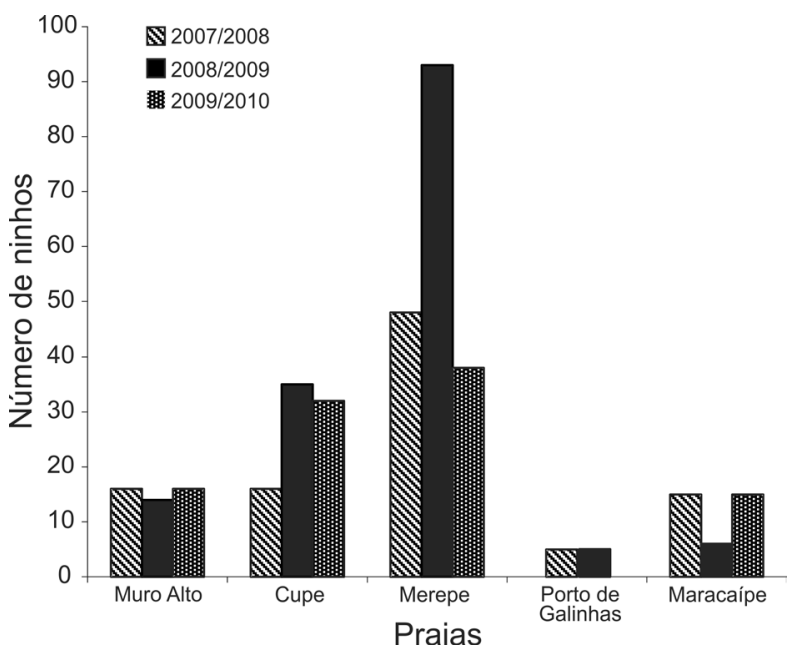

Fig. 5. Número de ninhos de Eretmochelys imbricata (Linnaeus, 1766) por praia registrados entre as temporadas reprodutivas de 2007 e 2010 nas praias do município de Ipojuca, PE, Brasil. A praia de Merepe apresentou o maior número de desovas nas três temporadas reprodutivas. ninhos das temporadas ocorreu em 2008/2009 na praia de Merepe, com 24 ninhos/km e as menores frequências registradas nas praias de Porto de Galinhas e Maracaípe (Tab. II).

Biologia reprodutiva. $O$ sucesso reprodutivo ou proporção de filhotes vivos foi de $65,6 \%$ para as temporadas. A taxa observada para as temporadas reprodutivas isoladamente foi de $59 \%, 68,6 \%$ e $45,9 \%$, respectivamente em 2007/2008, 2008/2009 e 2009/2010. O sucesso reprodutivo foi significativamente diferente de acordo com o teste Kruskal-Wallis $\left(X^{2}=19.3023\right.$, df $=2, \mathrm{p}$-valor $=0,043)$.

Foram protegidos ao todo 29.735 filhotes de tartarugas de pente. A média do número de filhotes foi de $87,8 \pm 39,6$ em 2007/2008, 92,3 $\pm 30,1$ em 2008/2009 e $66,13 \pm 54$ em 2009/2010.

A média e desvio padrão da fecundidade registrada para $E$. imbricata foi de $147 \pm 28$ ovos $(\mathrm{N}=100$, 69-206) em 2007/2008, 142 \pm 36 ovos ( $\mathrm{N}=153,77-212)$ em 2008/2009 e de 153 $\pm 53,5$ ovos em 2009/2010 $(\mathrm{N}=103$, 75-227). Observou-se que as temporadas reprodutivas foram significativamente diferentes quanto à fecundidade $\left(\mathrm{X}^{2}=10.004, \mathrm{df}=2, \mathrm{p}\right.$-valor $\left.=0.006725\right)$. Foi analisada a relação entre a fecundidade (número de ovos por ninho) e o CCC das fêmeas. Por meio de regressão linear observou-se que as variáveis fecundidade e comprimento não estão fortemente relacionadas $\left(\mathrm{R}^{2}=\right.$ 0,1592) (Fig. 6).

Foram obtidas medidas biométricas de 59 fêmeas no momento da desova, sendo sete tartarugas em 2007/2008, 25 em 2008/2009 e 27 em 2009/2010. Registrou-se a média e o desvio padrão para o CCC de 92,5 $\pm 4,5$ (73-115), e para a LCC de 83,4 \pm 5 (71-106).

Testes de correlação revelaram que as medidas do CCC e LCC da fêmea em idade reprodutiva não apresentaram correlação com as variáveis número de filhotes, ovos totais e natimortos.

A duração média do tempo de incubação foi de 55 dias (40-67, N=201). Houve diferença significativa

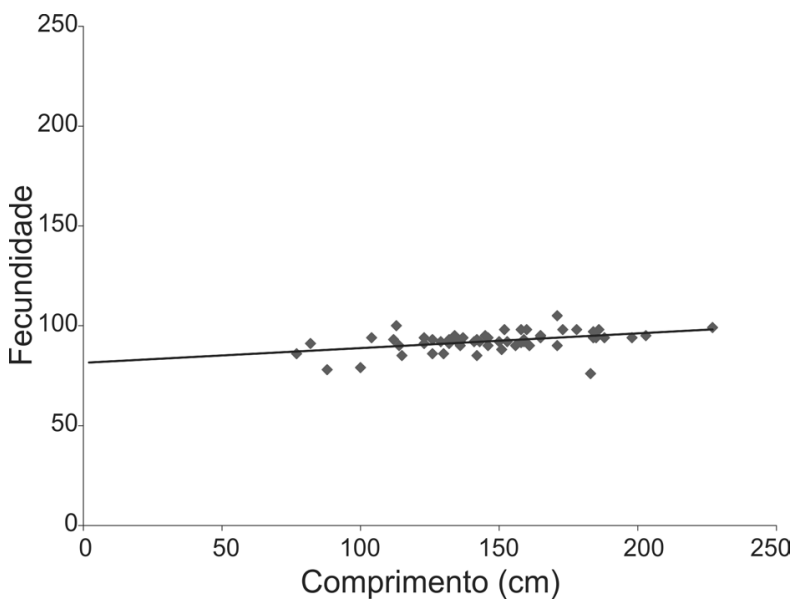

Fig. 6. Regressão linear entre fecundidade das fêmeas (número de ovos) e comprimento curvilíneo da carapaça das fêmeas de Eretmochelys imbricata (Linnaeus, 1766) nas praias de Ipojuca, PE, Brasil. Expressão da equação: $\mathrm{x}=80.04+0.08 \mathrm{y} ; \mathrm{R}^{2}=0.1592$. 
Tab. I. Distribuição dos ninhos de Eretmochelys imbricata (Linnaeus, 1766) nas praias do Ipojuca, PE, Brasil durante três temporadas reprodutivas.

\begin{tabular}{ccccc}
\hline Temporadas reprodutivas & Muro Alto & Cupe & Merepe & Porto de Galinhas \\
\hline $2007 / 2008$ & 16 & 16 & 48 & 5 \\
$2008 / 2009$ & 14 & 35 & 32 & 5 \\
$2009 / 2010$ & 16 & 38 & 15 & 0 \\
Total & 46 & 83 & 179 & 10 \\
\hline
\end{tabular}

Tab. II. Frequência de ninhos de Eretmochelys imbricata (Linnaeus, 1766) por praia nas temporadas reprodutivas de 2007 a 2010.

\begin{tabular}{|c|c|c|c|c|c|}
\hline Praias/ temporadas & Muro Alto & Merepe & Cupe & Porto de Galinhas & Maracaípe \\
\hline $2007 / 2008$ & 14 ninhos $/ \mathrm{km}$ & 12 ninhos $/ \mathrm{km}$ & 7 ninhos $/ \mathrm{km}$ & 3 ninhos $/ \mathrm{km}$ & 5 ninhos $/ \mathrm{km}$ \\
\hline $2008 / 2009$ & 12 ninhos $/ \mathrm{km}$ & 24 ninhos $/ \mathrm{km}$ & 15 ninhos $/ \mathrm{km}$ & 4 ninhos $/ \mathrm{km}$ & 2 ninhos $/ \mathrm{km}$ \\
\hline $2009 / 2010$ & 14 ninhos $/ \mathrm{km}$ & 10 ninhos $/ \mathrm{km}$ & 14 ninhos $/ \mathrm{km}$ & 0 ninhos $/ \mathrm{km}$ & 5 ninhos $/ \mathrm{km}$ \\
\hline
\end{tabular}

entre as três temporadas reprodutivas ( $\mathrm{p}$-valor $=0,042$ ). Em 2007/2008 foi observada uma média de 54 dias; em 2008/2009 esta média foi de 56 dias $(48-67, \mathrm{~N}=72)$, e em 2009/2010 foi de 60 dias (51-64, N=37). De acordo com o método bootstrap com $95 \%$ de confiança, o intervalo para o tempo médio de incubação vai de 54 a 56 dias.

Foram registrados dois grupos que diferiram em relação à média da variável sucesso reprodutivo, onde um dos deles teve média muito baixa igual a 28 filhotes vivos, e a outra média igual a 109 filhotes vivos.

\section{DISCUSSÃO}

Cerca de $80 \%$ das desovas foram registradas nos meses com maiores temperaturas, de janeiro a março. Estes picos de nidificação também foram observados por Mascarenhas et al. (2003), CAmillo et al. (2009) e Marcovaldi et al. (2011) nos estados da Paraíba, Bahia, Sergipe e Rio Grande do Norte (Tab. III). Isto demonstra o padrão sazonal no período de nidificação de E. imbricata nas praias do nordeste brasileiro. Observou-se claramente uma preferência temporal da espécie para nidificação nas praias do Ipojuca no período de temperaturas mais altas (Fig.1). O maior índice de desovas coincide com um período de maior fluxo turístico na área, sendo por isso, essencial o monitoramento das tartarugas marinhas que ocorrem nas praias do Ipojuca, para garantir a proteção das fêmeas adultas, que saem do mar exclusivamente para desovar, bem como a preservação dos ninhos e a viabilidade dos ovos.

A utilização do modelo SARIMA é conveniente, pois a partir de dados das temporadas pode-se ter um padrão das variações do período de nidificação e isto pode auxiliar no trabalho de monitoramento de desovas, indicando situações onde o número de ninhos identificados seja menor do que o esperado, o que deve ser cuidadosamente investigado pela equipe responsável pelo monitoramento. Para SANTOS et al. (2010), as fêmeas em idade reprodutiva com maior massa corpórea e com bom estado corporal tendem a nidificar mais cedo. Portanto, também se faz necessário coletar dados de massa corpórea e energia utilizada na nidificação, no intuito de se explicar possíveis variações no período de desovas.

Tab. III. Aspectos relativos à biologia reprodutiva de Eretmochelys imbricata (Linnaeus, 1766) em diferentes locais de nidificação do Brasil.

\begin{tabular}{|c|c|c|c|c|}
\hline & Pernambuco & Bahia & Paraíba & Rio Grande do Norte \\
\hline Temporadas reprodutivas & 2007 a 2010 & 2004 a 2008 & 2002 a 2003 & 2006 a 2007 \\
\hline Período de nidificação & outubro a maio & outubro a abril & dezembro a maio & novembro a abril* \\
\hline Pico de desovas & $\begin{array}{l}\text { janeiro a março } \\
\qquad(>80 \%)\end{array}$ & $\begin{array}{l}\text { dezembro a fevereiro } \\
(>80 \%)^{*}\end{array}$ & fevereiro e março* & $\begin{array}{l}\text { janeiro a março } \\
\qquad(>80 \%)^{*}\end{array}$ \\
\hline Fecundidade média & 113,8 ovos/ninho & 136,4 ovos/ninho** & 150,6 ovos/ninho & - \\
\hline Densidade (ninhos/km) & 30 ninhos $/ \mathrm{km}$ & 26,2 ninhos $/ \mathrm{km}$ & 17,9 ninhos $/ \mathrm{km}$ & 26,5 ninhos $/ \mathrm{km}$ \\
\hline Sucesso reprodutivo & $65,6 \%$ & $78,3 \%$ & $79,8 \%$ & $57,6 \%$ \\
\hline $\begin{array}{l}\text { Média de filhotes vivos/ } \\
\text { ninho in situ }\end{array}$ & 77,7 filhotes/ninho & - & 120,11 filhotes/ninho & - \\
\hline CCC médio das fêmeas & $92,5 \mathrm{~cm}$ & $97,4 \mathrm{~cm}^{* *}$ & - & $83 \mathrm{~cm}$ a $103 \mathrm{~cm}$ \\
\hline LCC médio das fêmeas & $83,4 \mathrm{~cm}$ & $89,6 \mathrm{~cm}^{* *}$ & - & - \\
\hline $\begin{array}{l}\text { Tempo médio de } \\
\text { incubação dos ovos }\end{array}$ & 55 dias & 58,3 dias & - & 55 a 58 dias \\
\hline Fonte & Presente estudo & $\begin{array}{l}\text { Camillo et al., 2009; } \\
\text { *Marcovaldi et al., 2011; } \\
\text { **Marcovaldi et al., } 1999\end{array}$ & $\begin{array}{l}\text { *Mascarenhas et al., } \\
\text { 2003; } \\
\text { Mascarenhas et al., } 2004\end{array}$ & $\begin{array}{c}\text { Santos, 2008; } \\
\text { *Marcovaldi et al., } 2007\end{array}$ \\
\hline
\end{tabular}


Quanto à variação do número de ninhos de cada temporada, esta diferença pode estar relacionada às variações naturais da população (CHALOUPKA \& MusıcK, 1997), e ao intervalo de remigração (tempo entre as sucessivas temporadas, espécie-específico), comum entre as tartarugas marinhas (MilleR, 1997). Tal fenômeno já observado em E. imbricata por HiRTH (1980), sendo de 2,5-2,7 anos.

No que se refere à distribuição espacial dos ninhos, a escolha do local de desova pela fêmea é influenciada por vários fatores. As praias que apresentaram menor número de ninhos (Porto de Galinhas e Maracaípe) são caracterizadas por apresentarem ampla faixa de recifes de arenito, que dificulta o acesso das fêmeas até a praia para nidificar (Mortimer, 1990). Além disso, Porto de Galinhas é a praia de maior fluxo turístico, ocupação desordenada (Mortimer, 1990; Bugoni et al., 2001; SHANKER et al., 2004), e muita iluminação artificial (Witherington, 1992), fatores que também podem inibir a escolha desta praia como local de desova.

Por outro lado, as praias que tiveram maior número de ninhos (Merepe e Cupe) possuem menor declividade e considerável área coberta por vegetação de restinga. Segundo SERAFIni et al. (2009), acima de $50 \%$ das desovas de E. imbricata ocorrem em áreas cobertas ou próximas à vegetação. No presente estudo, notou-se que houve um registro diferencial em relação à distribuição espacial dos ninhos de E. imbricata nas praias do município do Ipojuca durante as temporadas reprodutivas analisadas, com preferência pelas praias abertas, desprovidas de recifes, ampla cobertura vegetal e com menor influência antrópica.

De acordo com FERREIRA JR (2009), o registro de tartarugas marinhas estaria relacionado com regiões de adensamento vegetal, onde a taxa de predação é menor. Outro fator que favorece a escolha do local para desova são praias que não apresentam barreiras físicas como recifes (MorTimer, 1990). Neste caso, Merepe parece apresentar todas as características favoráveis à nidificação.

Para justificar com precisão a preferência das tartarugas por algumas praias, é necessário coletar dados abióticos como granulometria (FERREIRA JR, 2009), inclinação (FERreIRA JR et al., 2003), e bióticos como taxa de cobertura vegetal (LÓPEZ-MENDILAHARSU \& RochA, 2009) e outras variáveis que caracterizam as praias. Assim, através de análises estatísticas é possível verificar o comportamento de distribuição da espécie associado a esses parâmetros.

O sucesso reprodutivo encontrado neste estudo está de acordo com os valores observados para a espécie no Brasil (Gomes et al., 2007). Foi observado que houve uma redução da proporção de filhotes vivos na última temporada estudada, e que pode estar relacionada às variações climáticas no ambiente de incubação (AcKerman, 1997; Ferreira JR, 2009). Outro aspecto que atua na determinação do sucesso reprodutivo é o nível de variabilidade genética da população; este fator também deve ser avaliado para se inferir acerca da conservação desta espécie, pois populações com maiores níveis de variabilidade genética são mais resistentes às intempéries ambientais que tanto influenciam no desenvolvimento destes animais (JosEPH \& SHAW, 2011).

A relação entre fecundidade e CCC das fêmeas, apesar de ter sido positiva, neste estudo não foi significativamente relevante, como já observado por MARCOVAldi et al. (1999) e XAVIER et al. (2006).

Não houve correlação entre as variáveis biométricas das fêmeas com número de filhotes, ovos totais e natimortos. Essas últimas variáveis estão relacionadas às condições ambientais do ninho, principalmente com a temperatura (FERREIRA JR, 2009), tornando evidente a importância da preservação dos locais de desova, focando áreas que estão suscetíveis à ação antrópica (BuGONi et al., 2001).

O tempo de incubação de cada ninho é contabilizado a partir do dia em que ocorreu a desova até o dia da eclosão dos filhotes. Este período pode variar dependendo de alguns fatores abióticos como as variações de temperatura do ambiente (ACKERMAN, 1997), pluviosidade, umidade relativa, granulometria da areia (ACKerman, 1997; Ferreira JR et al., 2003), concentração de $\mathrm{CO}_{2}$ e $\mathrm{O}_{2}$ (ACKERMAN, 1997; FERREIRA $\mathrm{J}_{\mathrm{R}}, 2009$ ) e outros fatores que atuam diretamente no metabolismo dos neonatos, acelerando ou retardando o tempo necessário para o desenvolvimento dos filhotes (ACKERMAN, 1997). Os valores encontrados para as praias do Ipojuca corroboram valores relatados por CAmillo et al. (2009) na Bahia e SANTos et al. (2010) no Rio Grande do Norte. Os percentis da distribuição empírica de bootstrap para o tempo de incubação de $E$. imbricata permitem que os pesquisadores possam ter uma quantificação sobre os tempos mínimos e máximos de eclosão, aumentando assim as chances de flagrar o nascimento dos filhotes e coletar dados necessários para pesquisas científicas.

Os grupos encontrados através do agrupamento Kmeans devem ser relacionados às diferentes características das praias. Entretanto, essa relação deverá ser acessada por meio de análises multivariadas com diferentes variáveis específicas do microambiente do ninho de cada praia, tais como umidade, concentração de gases, granulometria e composição dos sedimentos, temperatura e pluviosidade (ACKERMAN, 1997).

Agradecimentos. À ONG Ecoassociados, pelo suporte técnico.

\section{REFERÊNCIAS BIBLIOGRÁFICAS}

Ackerman, R. A. 1997. The Nest Environment and the Embryonic Development of Sea Turtles. In: Lutz, P. L. \& Musick, J. A. eds. The Biology of Sea Turtles. Boca Raton, CRC Press. p.83-103.

Alvarado, J. \& Murphy, T. M. 1999. Nesting Periodicity and Internesting Behavior. In: EcKert, K. L.; BJorndal, K. A.; ABreUGrobois, F. A. \& Donnelly, M. eds. Research and Management 
Techniques for the Conservation of Sea Turtles. Pennsylvania, IUCN/SSC Marine Turtle Specialist Group. 248p.

Anjos, A.; Mazza, M. C. M.; Santos, A. C. M. C. \& Delfini, L. T. 2004. Análise do padrão de distribuição espacial da Araucária (Arancaria angustifolia) em algumas áreas do estado do Paraná, utilizando a função K de Ripley. Scientia Forestalis 66:38-45.

Bolten, A. C. 1999. Techniques for Measuring Sea Turtles. In: EcKert, K. L.; BJorndal, K. A.; Abreu-Grobois, F. A. \& Donnelly, M. eds. Research and Management Techniques for the Conservation of Sea Turtles. Pennsylvania, IUCN/SSC Marine Turtle Specialist Group. $248 \mathrm{p}$.

Bugoni, L.; Krause, L. \& Petry, M. V. 2001. Marine Debris and Human Impacts on Sea Turtles in Southern Brazil. Marine Pollution Bulletin 42(12):1330-1334.

Camillo, C. S.; Romero, R. M.; Leone, L. G.; Batista, R. L. G.; Velozo, R. S. \& Nogueira-Filho, S. L. G. 2009. Características da reprodução de tartarugas marinhas (Testudines, Cheloniidae) no litoral sul da Bahia, Brasil. Biota Neotropica 9(2).

ChaloupKa, M. Y. \& Musick, J. A. 1997. Age, Growth, Population Dynamics. In: Lutz, P. L. \& Musick, J. A. eds. The Biology of Sea Turtles. Boca Raton, CRC Press. p.233-270.

CITES - Conventional On International Trade In Endangered Species of Wild Fauna and Flora. 2011. Species data base. Disponível em: $<\mathrm{http}: / /$ www.cites.org/eng/resources/species.html $>$. Acesso em: 20.02.2011.

Davison, A. C. \& HinckLey, D. 1997. Bootstrap Methods and their Application. Cambridge, Cambridge Series in Statistical and Probabilistic Mathematics. 582p.

FERreira JR, P. D. 2009. Efeitos de Fatores Ambientais na Reprodução de Tartarugas. Acta Amazonica 39(2):319-334.

Ferreira JR, S. N. M.; Castro, P. de T. A.; Addad, J. E. \& Lorenzo; M. 2003. Aspectos fisiográficos das áreas de nidificação da tartaruga marinha Caretta caretta na praia da Guanabara, Anchieta, Espírito Santo, Arujá, SP. Anais do Instituto Pau Brasil de História Natural 7(1):25-40.

Gomes, M. G. T.; Santos, M. R. D. \& Henry, M. 2007. Tartarugas marinhas de ocorrência no Brasil: hábitos e aspectos da biologia da reprodução. Revista Brasileira de Reprodução Animal 30(1/2):19-27.

Hartigan, J. A. \& Wong, M. A. 1979. Algorithm AS 136: A k-means clustering algorithm. Applied Statistics 28(1):100-108.

HiRTH, H. F. 1980. Some aspects of the nesting behaviour and reproductive biology of sea turtles. American Zoologist 20:507523.

IBAMA - Instituto Brasileiro do Meio Ambiente e dos Recursos Renováveis. 2003. Lista nacional das espécies da fauna brasileira ameaçadas de extinção. Disponível em: $<$ http://www. ibama.gov.br>. Acesso em: 15.09.2010.

IUCN - International Union for Conservation of Nature. 2010. Red List of Threatened Species. Disponível em: <http://www.iucnredlist. org/apps/redlist/details/8005/0>. Acesso em: 28.11.2010.

Joseph, J. \& ShaW, P. W. 2011. Multiple paternity in egg clutches of hawksbill turtles (Eretmochelys imbricata). Conservation Genetics 12:601-605.

KRUSKAL, W. H \& Wallis, W. A. 1952. Use of ranks in one-criterion variance analysis. Journal of the American Statistical Association 47(260):583-621.

López-Mendilaharsu, M. \& Rocha, C. F. D. 2009. Comportamento de movimentação horizontal e vertical da tartaruga-de-couro Dermochelys coriacea. Oecologia Brasiliensis 13(1):99-114.

Marcovaldi, M. A.; Lopez, G. L.; Santos, A. J. B.; Bellini, C.; Santos, A. S. \& Lopez, M. 2011. Avaliação do Estado de Conservação da Tartaruga Marinha Eretmochelys imbricata (Linnaeus, 1766) no Brasil. Biodiversidade Brasileira 1:20-27

Marcovaldi, M. A. \& Marcovaldi, G. G. 1985. Projeto Tamar: área de desova, ocorrência e distribuição das espécies, época de reprodução, comportamento de postura e técnicas de conservação das tartarugas marinhas no Brasil. Brasília, MAIBDF. 46p.

1999. Marine turtles of Brazil: the history and structure of Projeto TAMAR-IBAMA. Biological Conservation 91(1):35-41. Marcovaldi, M. A.; Vieitas, C. S. \& Godfrey, M. H. 1999. Nesting and Conservation Management of Hawksbill Turtles (Eretmochelys imbricata) in Northern Bahia, Brazil. Chelonian Conservation and Biology 3(2):301-307.

Mascarenhas, R.; Zeppelin, D. F. \& Moreira, V. F. 2003. Observations on Sea Turtles in the State of Paraíba, Brazil. Marine Turtle Newsletter 101:16-18.

Miller, J. D. 1997. Reproduction in sea turtles. In: Lutz, P. L.; Musick, J. A. \& Wyneken, J. eds. The Biology of Sea Turtles. CRC Marine Science Series. Boca Raton, CRC Press. p. 51-71.

Mortimer, J. A. 1990. The influence of beach sand characteristics on the nesting behaviour and clutch survival of green turtles, Chelonia mydas. Copeia 3:802-817.

Packard, G. C.; Packard, M. J.; Miller, K. \& Boardman, T. J. 1987. Influence of moisture, temperature, and substrate on snapping turtle eggs and embryos. Ecology 4(68):983-993.

Pritchard, P. C. H. \& Mortimer, J. A. 1999. Taxonomy, External Morphology, and Species Identification. In: ECKERT, K. L.; Buorndal, K. A.; Abreu-Grobois, F. A. \& Donnelly, M. eds. Research and Management Techniques for the Conservation of Sea Turtles. Pennsylvania, IUCN/SSC Marine Turtle Specialist Group. 248p.

Raphael, B. L. 2003. Chelonians. In: Fowler, M. E. \& Miller, R. E. eds. Zoo and wild animal medicine. 5ed. Philadelphia, Saunders. p. $48-58$.

R Development Core Team. 2009. R: A language and environment for statistical computing. Vienna, R Foundation for Statistical Computing. Disponível em: $<$ http://www.R-project.org $>$. Acesso em: 10.08.2010.

Santana, W. M.; Silva-Leite, R. R.; Silva, K. P. \& Machado, R. A. 2009. Primeiro registro de nidificação de tartarugas marinhas das espécies Eretmochelys imbricata (Linnaeus, 1766) e Lepidochelys olivacea (Eschscholtz, 1829), na região da Área de Proteção Ambiental Delta do Parnaíba, Piauí, Brasil. Pan-American Journal of Aquatic Sciences 3(4):369-371.

Santos, A. J. B.; Freire, E. M. X.; Bellini, C. \& Corso, G. 2010 Body Mass and the Energy Budget of Gravid Hawksbill Turtles (Eretmochelys imbricata) during the Nesting Season. Journal of Herpetology 44:352-359.

Serafini, T. Z.; Lopez, G. G. \& Rocha, P. L. B. 2009. Nest site selection and hatching success of hawksbilland loggerhead sea turtles (Testudines, Cheloniidae) at Arembepe Beach, northeastern Brazil. Phyllomedusa 8(1):3-17.

Shanker, K.; Ramadevi, J.; Choudhury, B. C.; Singh, L. \& Aggarwal, K. 2004. Phylogeography of olive ridley turtles (Lepidochelys olivacea) on the east coast of India: implications for conservation theory. Molecular Ecology 13(7):1899-1909.

SpotiLA, J. R. 2004. Sea Turtles: a complete guide to their biology, behavior, and conservation. Baltimore, Johns Hopkins University Press, Oakwood Arts. 240p.

Werner, L. \& Ribeiro, J. L. D. 2003. Demand forecasting: an application of the Box-Jenkins models in the technical assistance of personal computer. Gestão e Produção 10(1):47-67.

Witherington, B. E. 1992. Behavioral Responses of Nesting Sea Turtles to Artificial Lighting. Herpetologica 48(1):31-39

WyNEKEN, J. 2001. The anatomy of sea turtles. Miami, NOAA. 180p.

Xavier, R.; Barata, A.; Cortez, L. P.; Queiroz, N. \& Cuevas, E. 2006. Hawksbill turtle (Eretmochelys imbricata Linnaeus, 1766) and green turtle (Chelonia mydas Linnaeus, 1754) nesting activity (2002-2004) at El Cuyo beach, Mexico. Amphibia-Reptilia 27:539-547.

Recebido em 08 de abril de 2011. Aceito em 25 de julho de 2012. ISSN 0073-4721

Artigo disponível em: www.scielo.br/isz 